\title{
Evidence regarding lingual fixed orthodontic appliances' therapeutic and adverse effects is insufficient
}

\author{
Abstracted from \\ Papageorgiou SN, Gölz L, Jäger A, Eliades T, Bourauel C. \\ Lingual vs. labial fixed orthodontic appliances: systematic review and \\ meta-analysis of treatment effects. Eur J Oral Sci 2016; 124: 105-118. \\ Address for correspondence: Spyridon N. Papageorgiou, Department of Orthodontics, School of Dentistry, \\ University of Bonn, Welschnonnenstr. 17, 53111 Bonn, Germany. E-mail: snpapage@gmail.com
}

\section{Question: In orthodontic patients, what are the treatment effects of lingual compared to labial fixed appliances?}

Data sources Medline, Cochrane Database of Systematic Reviews, Database of Abstracts of Reviews of Effects, Cochrane Central Register of Controlled Trials, Virtual Health Library and Web of Science were systematically searched up to July 2015 without limitations. Scopus, Google Scholar, ClinicalTrials.gov, the ISRCTN registry as well as reference lists of the trials included and relevant reviews were manually searched.

Study selection Randomised (RCTs) and prospective non-randomised clinical trials (non-RCTs) on human patients that compared therapeutic and adverse effects of lingual and labial appliances were considered. One reviewer initially screened titles and subsequently two reviewers independently screened the selected abstracts and full texts.

Data extraction and synthesis The data were extracted independently by the reviewers. Missing or unclear information, ongoing trials and raw data from split-mouth trials were requested from the authors of the trials. The quality of the included trials and potential bias across studies were assessed using Cochrane's risk of bias tool and the Grading of Recommendations Assessment, Development and Evaluation (GRADE) approach. For parallel trials, mean difference (MD) and the relative risk (RR) were used for continuous (objective speech performance, subjective speech performance, intercanine width, intermolar width and sagittal anchorage loss) and binary outcomes (eating difficulty), respectively. The standardised mean difference (SMD) was chosen to pool, after conversion, the outcome (oral discomfort) that assessed both binary and continuous. Random-effects meta-analyses were conducted, followed by subgroup and sensitivity analyses.

Results Thirteen papers pertaining to 11 clinical trials (three parallel RCTs, one split-mouth RCT and seven parallel prospective non-RCTs) were included with a total of 407 (34\% male/66\% female) patients. All trials had at least one bias domain at high risk of bias. Compared with labial appliances, lingual appliances were associated with increased overall oral discomfort, increased speech impediment (measured using auditory analysis), worse speech performance assessed by laypersons, increased eating difficulty and decreased intermolar width. On the other hand, lingual appliances were associated with increased intercanine width and significantly decreased anchorage loss of the maxillary first molar during space closure. However, the quality of all analyses included was judged as very low because of the high risk of bias of the included trials, inconsistency and imprecision. Conclusions Based on existing trials there is insufficient evidence to make robust recommendations for lingual fixed orthodontic appliances regarding their therapeutic or adverse effects, as the quality of evidence was low.

\section{Commentary}

More adult patients interested in orthodontic treatment led to the introduction of lingual fixed orthodontic appliances as an alternative to the unaesthetic appearance of conventional labial appliances. ${ }^{1,2,3}$ The design, manufacturing and mechanotherapy of the lingual appliances have seen constant improvement ${ }^{4}$, 5, 6, 7 alleviating some of their prior stigma for poorer outcomes, insertion/handling difficulties and higher lab costs and longer chairtime. ${ }^{8}$ Recently, it has been claimed that lingual appliances may provide less noticeability, anchorage loss, forces applied, white spot lesions and caries. 2, 9, 10 In addition, keeping up to date with the most recent evidence is increasingly challenging for clinicians. ${ }^{11,} 12$ Therefore, the objective of the study of Papageorgiou et al. was to compare the effects of lingual and labial appliances from clinical trials in orthodontic patients using an evidence-based approach.

The authors registered their systematic review protocol in PROSPERO. ${ }^{13}$ The review question, inclusion criteria and search strategy were clearly described and adequately addressed as per the Cochrane Handbook ${ }^{14}$ and the PRISMA statement. ${ }^{15}$ The review authors compared treatment effects of lingual appliances with labial appliances from the correct clinical studies design (prospective). However, various limitations were noted. Most of the included trials examined short-term adverse effects and had mostly unclear and high risk of bias. Nine trials had two or less domains categorised as low risk of bias. The inadequate or non-existent randomisation and lack of blinding of the outcome assessors were the most problematic domains. Only four out of the 11 included trials in this review were RCTs. The limited number of RCTs could affect the review with the principle of 'garbage in, garbage out.' ${ }^{16}$ As was shown in the sensitivity analyses performed, this might influence the magnitude and direction of the observed treatment effect estimates. ${ }^{17}$ Two of RCTs $^{18,19}$ were considered the superior trials since they had low risk of bias in most (4/7) of the assessed domains. Overall, the sample size (range: RCTs, 20-52; non-RCTs, 12-60) of the included trials evaluating each outcome was small. All results call for caution as the GRADE score ${ }^{20}$ for these meta-analyses was very low to moderate. The sensitivity analysis considered only the RCTs initially. The 
advantage of performing the sensitivity analysis is that factors negative to the quality of the clinical recommendations are possible to identify. ${ }^{21}$

Papageorgiou et al. report that the overall evaluation of RCTs and non-RCTs indicates that a lack of randomisation had a significantly beneficial effect for lingual appliances compared with the RCTs. Conversely, the table (Table S8) that supports this information was missing from the supplementary section.

This review produced new information compared to the two previous SRs that ask a similar clinical question, however they further included retrospective studies ${ }^{22}$ or had limited trials and lacked the GRADE approach. ${ }^{8}$

With the following statement the authors summarised the limitations of this review to guide clinical decision-making: 'There is insufficient evidence at present to make robust recommendations for lingual fixed orthodontic fixed appliances regarding their therapeutic or adverse effects. Only two of the 11 trials identified were randomized, and none was at low risk of bias.' 23 For example, for heterogeneity and subgroup analyses, the Knapp-Hartung adjustment ${ }^{24}$ requires that at least five trials be pooled. However, the oral discomfort and the objective speech performance outcomes, which contained the most trials, had only three each.

From the findings based on this moderate quality of evidence (estimated from the sensitivity analysis), patients may present more oral discomfort, speech impairment and eating difficulty with lingual appliances compared with labial appliances. However, the very low quality of this evidence prevents one from making strong recommendations about the effects of lingual appliances. As such, orthodontists and patients must consider this as cause for caution in their clinical decision-making process.

There is therefore a need for high quality, long-term parallel randomised controlled trials comparing lingual and labial appliances in order to provide strong clinical recommendations in the future. Papageorgiou et al. accurately suggest that these trials should primarily focus on objective measurements of therapeutic or adverse effects and follow the CONSORT statement. ${ }^{25}$

Kelvin I Afrashtehfar McGill University Faculty of Dentistry, Montreal, Canada
1. Gorman JC. Treatment of adults with lingual orthodontic appliances. Dent Clin North Am 1988; 32: 589-620.

2. McDonald F, Cobourne M. Adult orthodontics: perils and pitfalls. Prog Orthod 2007; 8: $308-313$.

3. Afrashtehfar Kl, Assery MK. Five considerations in cosmetic and esthetic dentistry. I N J Dent Assoc 2014; 85: 14-15.

4. Wiechmann D. Lingual orthodontics (part 1): laboratory procedure. J Orofac Orthop 1999; 60: 371-379

5. Wiechmann D. Lingual orthodontics (part 2): archwire fabrication. J Orofac Orthop 1999; 60: 416-426.

6. Grauer D, Proffit WR. Accuracy in tooth positioning with a fully customized lingual orthodontic appliance. Am / Orthod Dentofacial Orthop 2011; 140: 433-443.

7. Fukawa R. Lingual orthodontics in the new era treatment according to criteria for occlusion and aesthetics. Int Orthod 2009; 7: 370-402.

8. Long $\mathrm{H}$, Zhou $\mathrm{Y}$, Pyakurel $\mathrm{U}$, et al. Comparison of adverse effects between lingual and labial orthodontic treatment. Angle Orthod 2013; 83: 1066-1073.

9. Lawson RB. Extraction treatment in lingual orthodontics. / Orthod 2013; 40: S38-48.

10. Dalessandri D. Reduction in incidence of white spot lesions with lingual appliances. Am J Orthod Dentofacial Orthop 2016; 149: 7-8

11. Afrashtehfar KI, Eimar H, Yassine R, Abi-Nader S, Tamimi F. Evidence-based dentistry for planning restorative treatments: barriers and potential solutions. Eur J Dent Educ 2016; doi: 10.1111/eje.12208. [Epub ahead of print]

12. Richards D. Keeping up to date. Evid Based Dent 2015; 16: 98

13. Papageorgiou SN, Gölz L, Eliades T, Bourauel C. Comparison of the therapeutic effects and adverse effects of lingual versus labial fixed orthodontic appliances: a systematic review and meta-analysis. PROSPERO 2015: CRD42015024596. Available from http://www.crd.york.ac.uk/PROSPERO/display record.asp?ID=CRD42015024596

14. Higgins ], Green S, The Cochrane Collaboration. Cochrane Handbook for Systematic Reviews of Interventions Version 5.0.2. S.I.: The Cochrane Collaboration; 2008. p. 1 online resource.

15. Moher D, Liberati A, Tetzlaff J, Altman DG; PRISMA Group. Preferred reporting items for systematic reviews and meta-analyses: the PRISMA statement. / Clin Epidemiol 2009; 62: 1006-1012.

16. Yu XP, Xu L, Yu RQ. [Not Available]. . Autom Methods Manage Chem 2009; 2009: 291820.

17. Papageorgiou SN, Antonoglou GN, Tsiranidou E, Jepsen S, Jager A. Bias and smallstudy effects influence treatment effect estimates: a meta-epidemiological study in oral medicine. J Clin Epidemiol 2014; 67: 984-992.

18. Khattab TZ, Farah H, Al-Sabbagh R, Hajeer MY, Haj-Hamed Y. Speech performance and oral impairments with lingual and labial orthodontic appliances in the first stage of fixed treatment. Angle Orthod 2013; 83: 519-526.

19. Khattab TZ, Hajeer MY, Farah H, Al-Sabbagh R. Maxillary dental arch changes following the leveling and alignment stage with lingual and labial orthodontic appliances: a preliminary report of a randomized controlled trial. / Contemp Dent Pract 2014: 15: 561-566.

20. Guyatt GH, Oxman AD, Schunemann HJ, Tugwell P, Knottnerus A. GRADE guidelines: a new series of articles in the Journal of Clinical Epidemiology. I Clin Epidemiol 2011; 64: 380-382.

21. Donegan S, Williamson P, Gamble C, Tudur-Smith C. Indirect comparisons: a review of reporting and methodological quality. PLoS One 2010; 5: e11054.

22. Mistakidis I, Katib H, Vasilakos G, Kloukos D, Gkantidis N. Clinical outcomes of lingual orthodontic treatment: a systematic review. Eur J Orthod 2015; pii: cjv061. [Epub ahead of print]

23. Papageorgiou SN, Golz L, Jager A, Eliades T, Bourauel C. Lingual vs. labial fixed orthodontic appliances: systematic review and meta-analysis of treatment effects. Eur I Oral Sci 2016; 124: 105-118.

24. Knapp G, Hartung J. Improved tests for a random effects meta-regression with a single covariate. Stat Med 2003; 22: 2693-2710.

25. Pandis N, Fleming PS, Hopewell S, Altman DG. The CONSORT Statement: Application within and adaptations for orthodontic trials. Am J Orthod Dentofacial Orthop 2015; 147: 663-679.

Evidence-Based Dentistry (2016) 17, 54-55. doi:10.1038/sj.ebd.6401172 